\title{
Psychiatric training in Spain: is there a need for change?
}

\author{
Jorge Cervilla
}

Over the last 20 years the Spanish system for postgraduate education has been channelled, for all specialities including psychiatry, through the MIR Exam (Médico Interno Residente). This is an exam that all Licenciates in medicine and surgery (the Spanish equivalent for the British MBBS degree) can take after finishing their medical school training.

\section{Recruitment of trainees}

The MIR exam consists of a marathon $250 \mathrm{MCQ}$ test that includes all possible subjects relevant to medicine, from statistics and biophysics to cardiac surgery and nuclear medicine. This exam can be taken once a year only and about one in five of all candidates pass it on each occasion. This exam is the only means of access to a training post in Spain and, in fact, it is becoming the only possible way to get a job after medical school training. The Spanish Department of Health offers a variable number of available posts (about $\mathbf{4 0 0 0}$ per year for some 18000 candidates) and candidates are ranked after the exam taking into account their scores in the MCQ test and their medical school academic records to give an order number from first (best performer) to last (worst performer) of those taking the exam. This number allows you to choose, between the offered posts, the one of your preference that remains untaken following the choice of those ranked above you.

Psychiatric training posts are usually taken before other more unpopular specialities such as GP rotations or most non-clinical specialities. Once you enter a psychiatric training rotation you are bound to the same hospital for the rest of your training although in some rotations trainees do spend some time in other local settings such as out-patient clinics and day hospitals. Part of the first year of training, as with most specialities, is spent doing some casualty on-call work. The training time is four years after which trainees are issued with a certificate of Specialist Training that enables them to do both public or private practice in the capacity of fully qualified specialist. There is no formal mean of higher psychiatric training as such. Furthermore, and this is true of most other European Union countries, there is no formal division between different psychiatric specialities such as child psychiatry or old age psychiatry.

\section{Clinical training}

The system usually provides a good training base for psychiatric trainees but training in general is rather patchy across the country with no unified criteria. Thus, some centres based on university cities generally provide a better training scheme. The amount of clinical work also varies across centres but in general comprises both daytime work (from $8 \mathrm{am}$ to $3 \mathrm{pm}$ ) and a one-in-five on call rota. The trainee is usually allowed a day off after having been on call.

Additionally, the trainee is never alone in the hospital when on call and, in spite of being left with most of the burden and routine workload, a fully qualified psychiatrist is always around in the hospital for immediate discussion and decision making. This is very helpful in terms of both training and service delivery.

Psychiatry is heavily medicalised in Spain (psychiatrists do wear white coats) and is far more developed in terms of in-patient treatments than in community orientated services. However, admissions to Spanish psychiatric wards (most of them within general hospitals) are conspicuously shorter on average than in English hospitals and the lack of adequate external services is very much absorbed by the patient's family, which is generally highly supportive.

\section{Supervision and academia}

Supervision of trainees is usually informal and tends to follow a case-to-case approach. There are, however, some centres in which some specialists have formal supervision sessions with the trainees. Junior doctors are not attached to one consultant but tend to move within the psychiatric department and follow whoever does the ward round that day.

Many trainees have the chance to complete a PhD degree during their training period and research is encouraged constantly by supervisors. 
Publication in Spanish journals is usually easy to achieve. In spite of much research of dubious quality, the amount of work done by some centres is astonishing and it is everyday more common to find Spanish papers in good international journals, although this privilege is still reserved to a group of high quality research centres.

\section{Drawbacks and suggestions to improve}

The main drawback in Spanish Specialist training is the lack of adequate enforcing controls over training. After trainees have passed the MIR exam they are virtually assured a specialist certification after 4 years. There is no psychiatric exam to ensure the quality of training and, in spite of the high quality of Spanish postgraduate training, nobody actually checks on the trainee to make sure that they have acquired the adequate skills. Moreover, given that the MIR exam is merely an MCQ paper (although a very difficult one), the personal characteristics of the trainee are not taken into account in the selection process nor in the final phase of the training.

The author believes that a formal demonstration of the trainee's capacities (sumilar to the MRCPsych) is needed prior to issuing a Specialist Certificate. This would ensure not only the quality of training but also improve clinical standards. It would also be desirable to have a number of minimal recommendations or guidelines for the supervision of trainees that were followed by all training centres. This would avoid the current situation that allows big differences in training for doctors trained in different hospitals (but sometimes in the same city). The homogeneity of the British experience could provide a useful model to improve further the Spanish psychiatric training system.

Jorge A. Cervilla, Research Fellow and Honorary Senior Registrar in Psychiatry, Institute of Psychiatry, London 